Nloma 2021, 39(2)

Revista de Psicologia, Ciències de l'Eduació i de l'Esport

ISSN: 1138-3194

Facultat de Psicologia, Ciències de l'Educació i de l'Esport Blanquerna

Universitat Ramon Llull

\title{
Implicaciones de la Teoría de Usos y Gratificaciones en las prácticas mediadas por redes sociales en el ámbito educativo. Una revisión sistemática de la literatura
}

\author{
Raquel Gil-Fernández ${ }^{1} \&$ Diego Calderón-Garrido ${ }^{2}$ \\ ${ }^{1}$ Universidad Internacional de La Rioja, UNIR (España) \\ ${ }^{2}$ Universitat de Barcelona (España) \\ Recibido: 2021-6-2 \\ Aceptado: 2021-9-30 \\ https://doi.org/10.51698/aloma.2021.39.2.63-74
}

Implicaciones de la Teoría de Usos y Gratificaciones en las prácticas mediadas por redes sociales en el ámbito educativo. Una revisión sistemática de la literatura

\begin{abstract}
Las tensiones entre los diferentes agentes educativos y la sociedad han propiciado infinidad de cambios en relación con los recursos que se usan para gestionar las diferentes relaciones. En la actualidad, las redes sociales se presentan como un recurso más a las que docentes y discentes recurren por diversos motivos. La Teoría de Usos y Gratificaciones se presenta como un enfoque óptimo para conocer las motivaciones que tienen los usuarios. En este artículo se realiza un análisis de la literatura científica publicada en SCOPUS y JCR entre los años 2001 y 2020 sobre la aplicación de dicha teoría a las redes sociales en el ámbito educativo. Los resultados obtenidos muestran que los artículos se distribuyen en tres temáticas principales: investigación educativa, psicología educativa y comunicación o información educativa. Como conclusión, se ha detectado que la Teoría de Usos y Gratificaciones otorga una perspectiva crítica que expone, con mayor tendencia, las limitaciones de las redes sociales. Sin embargo, también se han observado propuestas para las buenas prácticas.
\end{abstract}

Palabras clave: Teoría de Uso y Gratificaciones; redes sociales; influencia motivacional; perfiles de uso; revisión sistemática.

Implications of the Uses and Gratifications Theory for social media-mediated practices in education. A systematic review of literature

Abstract. The tensions between the different educational agents and society have led to countless changes in the resources used in the management of the different relationships. Currently, social media are viewed as a resource to which teachers and students turn for various reasons. The Uses and Gratifications Theory has often been characterised as an optimal approach to the study of the motivations of social media users. This research consists of an analysis of the scientific literature published in SCOPUS and JCR between 2001 and 2020 on the application of this theory to social media in educational settings. The results show that the articles are distributed into three main thematic areas: educational research, educational psychology and communication/information. In conclusion, it has been found that the Uses and Gratifications Theory offers a critical perspective that more often than not tends to highlight the limitations of social media. However, proposals for good practice have also been noted.

Keywords: Uses and Gratifications Theory; social media; motivational influence; profiles of use; systematic review.

Correspondencia

Diego Calderón-Garrido

Serra Hunter Fellow

Universitat de Barcelona,

ORCID: https://orcid.org/0000-0002-2860-6747

Email: dcalderon@ub.edu 


\section{Introducción}

El paradigma educativo experimenta continuos cambios y modificaciones que son producto de las interacciones entre la sociedad y el ámbito formativo. En el terreno de la tecnología, el uso de redes sociales por parte de todos los agentes implicados en el proceso de enseñanza-aprendizaje y su interacción han sido objeto de estudio por parte de los investigadores, se ha ido intensificando y constituye una tendencia que está alcanzando gran peso en los últimos años.

En este contexto educativo, las redes sociales impulsan el constructivismo cognitivo y social -entendido como la interacción entre individuo y situación- de una manera holística, dado que por sus características intrínsecas proporcionan un espacio que permite a las personas comunicarse, colaborar, interactuar y compartir ideas (Saini \& Abraham, 2019). El establecimiento de entornos virtuales en las comunidades formativas y las experiencias mediadas por redes sociales han sido estudiadas utilizando diversos enfoques que dieran respuesta a los objetivos perseguidos. Uno de estos enfoques se basa en la aplicación de la Teoría de Usos y Gratificaciones (en adelante TU\&G)

La TU\&G fue formulada en la década de los cuarenta como respuesta a la preocupación por conocer los factores sociológicos y psicológicos que influían en que los usuarios adoptaran o rechazaran determinados medios de comunicación e información. De este modo, se estableció un modelo teórico válido para entender qué se escondía detrás de dicha elección (Saini \& Abraham, 2019). Katz (1959), tiempo después, continuó profundizando en la teoría y se basó en el cumplimiento de determinadas necesidades de los usuarios.

Davis (1986), basándose en la teoría de la acción razonada (como se cita en Park et al., 2007), formula el Modelo de Aceptación de Tecnología (TAM). Las debilidades de este modelo fueron percibidas por su propio autor (Davis, 1989), que descubrió que faltaban variables para detectar cuestiones como la utilidad y facilidad de uso percibida. Para buscar una mayor explicación contextual, complementó el modelo con el enfoque de la TU\&G (Katz et al., 1974).

La TU\&G posee una configuración muy versátil y se puede aplicar a todo tipo de medios y tecnologías, desde las más clásicas, como radio, televisión o prensa, a los medios contemporáneos (Chen, 2018). En la actualidad se aplica ampliamente a medios digitales, como Internet o las redes sociales. Se parte de la base de que los usuarios son agentes activos en cuanto a la elección y evaluación de los recursos mediáticos a su alcance y tomarán decisiones racionales para seleccionarlos según sus creencias sobre cuáles satisfarán mejor sus necesidades y maximizarán su gratificación personal. Los factores psicológicos y sociales influyen en esta toma de decisión racional (Leung \& Wei, 2000; Luo \& Remus, 2014). Pueden ser intrínsecos -se participa de determinado medio por placer- o extrínseco -se obtiene un beneficio- (Luo et al., 2011).
La TU\&G pronto se consolidó como un enfoque óptimo para explicar los factores determinantes de motivaciones, uso y continuidad de las redes sociales, y se comenzó a aplicar a este medio específico (Lee \& Ma, 2012). Hsu et al. (2015) advirtieron la existencia de ciertas variables que había que tener en cuenta: estos modelos teóricos no son de aplicación universal y hay que tener presentes algunos factores, como las características culturales, las actividades de los usuarios o el nivel sociocultural, ya que modelan la relación entre los usos y las gratificaciones de los consumidores. Estos autores ven en la TU\&G la repuesta a la detección de estos factores transformadores y motivacionales. Por todo lo descrito anteriormente, se puede aducir que es relevante aplicar la teoría a campos y poblaciones concretas para detectar particularidades y necesidades, como es el caso del ámbito de la educación.

Como se describirá en este trabajo, en el ámbito de la formación, la TU\&G sirve para identificar factores como la detección de oportunidades profesionales, la interacción social, la búsqueda y el intercambio de información profesional, y aspectos emocionales relacionados con la relajación y el disfrute en el contexto educativo (Dhir et al., 2017; Gallego et al., 2016; Saini \& Abraham, 2019).

Así pues, el objetivo de este artículo es realizar una revisión sistemática de la literatura científica de impacto publicada durante el periodo comprendido entre 2001 y 2020, centrada en las investigaciones que aplican la TU\&G a las redes sociales utilizadas en contextos educativos. Se pretende actualizar y reafirmar el conocimiento que se tiene sobre la TU\&G y su aplicación a las redes sociales, detectar la evolución de las tendencias en investigación sobre este particular, y describir y sintetizar de manera sistemática las evidencias aportadas por la literatura científica con respecto a la temática objeto del trabajo.

\section{Metodología y diseño de la investigación}

Para conseguir el objetivo propuesto, la búsqueda se concretó en la literatura científica que utilizaba la TU\&G desde alguna perspectiva, para determinar aspectos relacionados con el uso de las redes sociales en contextos educativos. Se procedió a realizar dicha búsqueda y a seleccionar y analizar los artículos según los criterios que se exponen a continuación.

\section{Parámetros generales de búsqueda de la literatura}

La generación y posterior publicación y difusión del conocimiento científico en cualquier ámbito es una tarea primordial del investigador, pero no es menos importante emprender estudios sobre temáticas, procedimientos, procesos de selección y criterios que hayan guiado esas publicaciones (Hernández-González et al., 2017). Las revisiones sistemáticas atienden a estos fines, y sintetizan la investigación a partir del análisis de los estudios primarios. Como se pretende en este trabajo, se trata de detectar los antecedentes de un tema 
en un ámbito escogido para determinar las directrices y tendencias presentes y futuras en la investigación de este, y configurar unas "hojas de ruta" para los investigadores (Morales et al., 2017)

En este caso, la búsqueda se centró en dos bases de datos de referencia para las ciencias sociales: Journal Citation Reports (JCR) y SCOPUS. Ambas se consideran excelentes indicadores de calidad de la literatura científica y proporcionan un enfoque multidisciplinar en los resultados. Por un lado, JCR pertenece, entre otras, a la Core Collection de la Web of Science (WoS), y está compuesta por dos subgrupos: la Science Citation Index (SCI) y la Social Sciences Citation Index (SSCI). Las instituciones académicas y los investigadores la reconocen como la mejor fuente de información en el campo científico, ya que las revistas que se encuentran alojadas en ella han experimentado rigurosos criterios de calidad para ser indexados. Los datos que JCR suministra orientan las políticas científicas y académicas (Pereira, 2018). Por otro lado esta investigación se completó con la búsqueda de literatura en SCOPUS dada la amplia gama de cobertura de estudios multidisciplinares que aloja esta base de datos.

El acceso se realizó a través del portal web de la Fundación Española para la Ciencia y la Tecnología (FECYT). Todo el proceso realizado, los resultados de las revisiones y los metaanálisis, siguieron las indicaciones del protocolo diseñado en el Preferred Reporting Items for Systematic Reviews and Meta-Analyses (PRISMA) (Hutton et al., 2016; Urrútia \& Bonfill, 2010) y se tuvieron en cuenta los parámetros metodológicos establecidos por Alexander (2020) para establecer la calidad de las revisiones sistemáticas.

\section{Proceso de búsqueda y selección de los trabajos}

La recolección documental se realizó, en primera instancia, entre los artículos publicados en revistas indexadas en la base de datos JCR, plataforma de la Web of Science, Core Collection. El período de tiempo seleccionado fueron los años 2001-2020. Si bien es cierto que la TU\&G se promulgó y desarrolló en la década de los cuarenta del siglo pasado, su aplicación a los usos educativos de redes sociales es reciente. Se consideró de interés comenzar el análisis en la década en la que se comenzó a extender el uso de las redes sociales, si bien no se obtuvieron trabajos que cumplieran con los parámetros de selección hasta 2011.

Para el análisis se procedió, en primer lugar, a la depuración de los datos que ofrece la propia plataforma al incluir descriptores. La combinación utilizada fue "education" AND "uses and gratifications" De este modo se podía acceder a los artículos que contuvieran dichos descriptores tanto en el título, como en el resumen o en las palabras clave. Se evitaron los tópicos, como "social media" o "social network" para no correr el riesgo de discriminar trabajos que se basaran en varios medios y el discurso transversal entre los mismos e incluyeran las redes sociales sin aparecer alusión a las mismas en el título, resumen o en las palabras clave. También se evitó utilizar el término teoría en los descriptores, dado que los autores en ocasiones utilizan los conceptos modelo o ley, pero no aclaran por qué eligen una terminología $\mathrm{u}$ otra.

En la Core Collection de Web of Science (SCI, SSCI, $A \& H C I, C P C I-S, C P C I-S S H, B K C I-S$ y ESCI) se publicaron un total de 56 trabajos que obedecían a los descriptores indicados. Al período 2001 a 2020 pertenecen 52. De estos, 28 se encontraban en la base de datos JCR (SCI y $S S C I$ ). No existió discriminación en cuanto a categorías de WoS (dado el carácter multidisciplinar del tema), ni al tipo de acceso o tipo de documento (si bien al aplicar los criterios de inclusión, en el resultado final únicamente quedaron artículos). Posteriormente, tras emplear las pautas que se indican más abajo, 16 trabajos pasaron a la fase de análisis.

En SCOPUS se emplearon los mismos tópicos. Se obtuvieron un total de 138 documentos, e igualmente no se estableció discriminación por materia, tipo de documento, idioma o procedencia geográfica. Tras seleccionar el período de tiempo escogido quedaron un total de 118. Finalmente, la muestra de análisis se redujo a 38 artículos.

Al cruzar los documentos obtenidos en ambas bases de datos y eliminar las repeticiones quedó una selección final de 39 trabajos. Los criterios de inclusión y exclusión son los que se exponen a continuación en la tabla 1 y en la figura 1.

\section{Análisis y categorización de los trabajos}

La revisión de las investigaciones se llevó a cabo en dos fases:

FASE 1. Cada uno de los autores del presente trabajo analizó de manera independiente los títulos, los resúmenes y las palabras clave de cada artículo para establecer las categorías principales. Posteriormente se reunieron para comparar y discutir las categorías básicas establecidas. Tras este primer análisis, finalmente se establecieron tres ámbitos de pertenencia de la literatura científica sobre el tema de estudio (ver tabla 2).

Tabla 1. Criterios de inclusión y exclusión

\begin{tabular}{|c|c|}
\hline Criterios de inclusión & Criterios de exclusión \\
\hline $\begin{array}{l}\text { Investigaciones sobre redes sociales } \\
\text { en el ámbito educativo que utilizan } \\
\text { la TU\&G en su desarrollo. Se } \\
\text { incluyen las que focalizan el } \\
\text { estudio en individuos que ejercen } \\
\text { cualquier rol educativo -aunque no } \\
\text { tengan intención didáctica } \\
\text { específica-y las que estudian } \\
\text { prácticas educativas en cualquier } \\
\text { tipo de población. }\end{array}$ & $\begin{array}{l}\text { Trabajos cuyo eje son otros media } \\
\text { y no contemplan el uso de medios } \\
\text { digitales }\end{array}$ \\
\hline $\begin{array}{l}\text { Nivel: todos los niveles educativos. } \\
\text { Educación formal, no formal e } \\
\text { informal. } \\
\text { Tipos de redes: redes sociales } \\
\text { genéricas y redes sociales } \\
\text { educativas, académicas o } \\
\text { profesionales. }\end{array}$ & $\begin{array}{l}\text { Trabajos que, utilizando medios } \\
\text { digitales, se refieren al uso de Internet } \\
\text { en general sin contemplar de manera } \\
\text { específica las redes sociales. }\end{array}$ \\
\hline $\begin{array}{l}\text { Áreas de conocimiento: ciencias } \\
\text { sociales y educación. } \\
\text { Idioma, tipo de fuente, tipo de } \\
\text { acceso: todos. }\end{array}$ & $\begin{array}{l}\text { Investigaciones cuya muestra no ha } \\
\text { sido recogida en ámbitos docentes o } \\
\text { que no tienen una intención } \\
\text { educativa. } \\
\text { Duplicaciones. }\end{array}$ \\
\hline
\end{tabular}




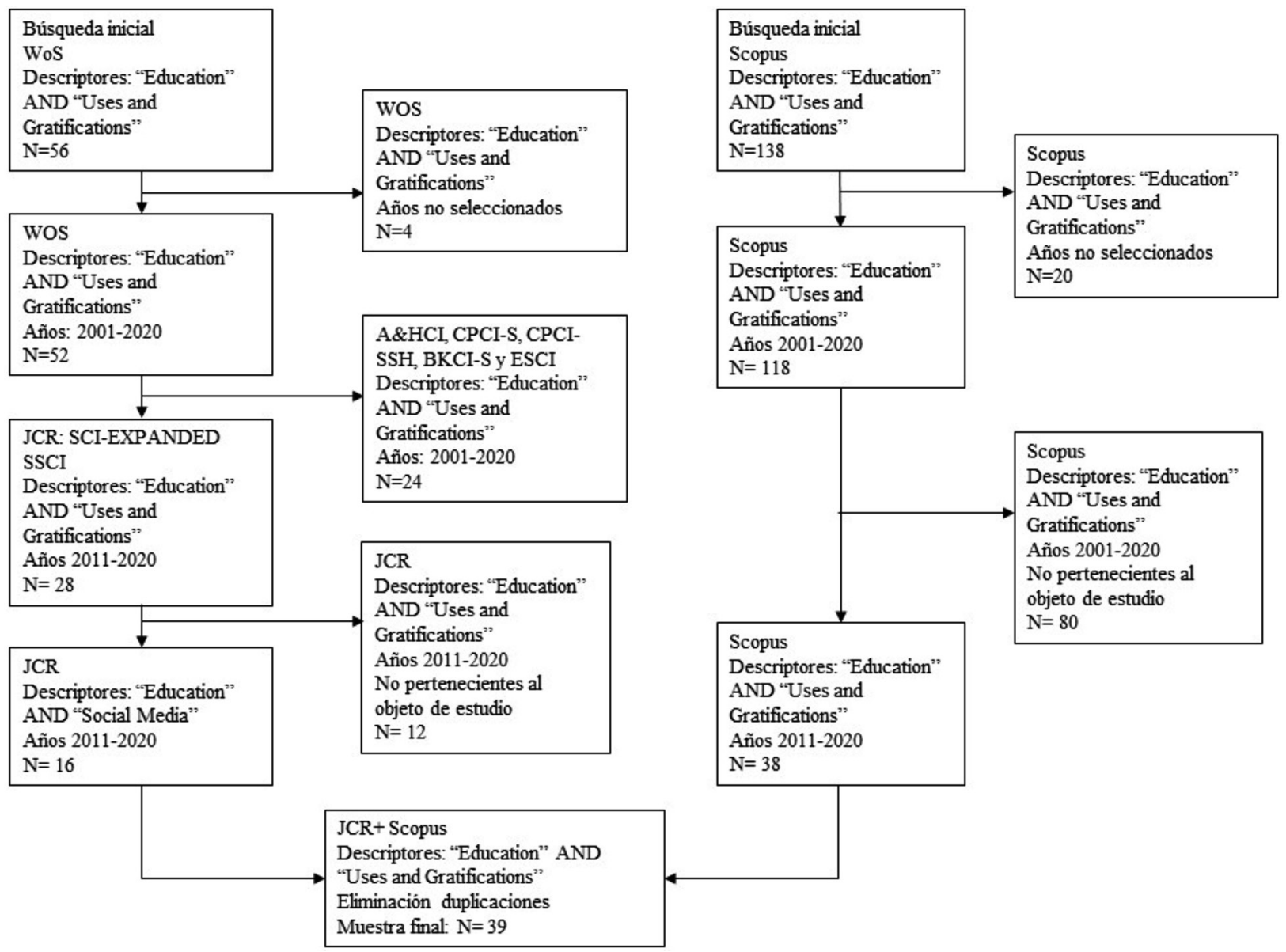

Figura 1. Diagrama de flujo de la selección de artículos realizada.

Tabla 2. Ámbitos principales de pertenencia

\begin{tabular}{ll}
\hline $\begin{array}{l}\text { Ámbitos de } \\
\text { pertenencia }\end{array}$ & Descripción \\
\hline Investigación educativa & $\begin{array}{l}\text { Trabajos que se basan en la búsqueda de motivaciones de uso a través de la TU\&G. Pueden tener un } \\
\text { carácter teórico o empírico. Se analizan, diseñan, establecen o implementan determinadas prácticas. } \\
\text { En ocasiones se toman decisiones inclusivas o se establecen programas. Los trabajos de esta categoría } \\
\text { se centran en los efectos, fines e implicaciones académicas. }\end{array}$ \\
\hline $\begin{array}{l}\text { Psicología educativa y } \\
\text { Psicopedagogía }\end{array}$ & $\begin{array}{l}\text { Trabajos que se centran en aspectos psicológicos de los actores más que en la red o experiencia que } \\
\text { media en el proceso. Aplican factores ligados a la personalidad del usuario según la TU\&G o } \\
\text { combinan varios modelos y marcos de la psicología con esta teoría. Se incluyen los trabajos que } \\
\text { buscan detectar prácticas y comportamientos negativos, compulsivos o poco seguros en el aula o en } \\
\text { individuos en formación fuera de la misma y los que detectan aspectos positivos para la satisfacción }\end{array}$ \\
\hline social de los individuos. \\
\hline $\begin{array}{l}\text { Procesos de comunicación e } \\
\text { información educativa }\end{array}$ & $\begin{array}{l}\text { Trabajos que se centran en cómo se produce en el mundo educativo el proceso de comunicación e } \\
\text { información mediado por redes sociales. En este grupo se incluyen los trabajos que detectan } \\
\text { diferencias socioculturales en el proceso comunicativo, así como actitudes como el emprendimiento, } \\
\text { la satisfacción personal o la seguridad en la comunicación. }\end{array}$ \\
\hline
\end{tabular}

Para la codificación se recurrió al software de análisis cualitativo Atlas.ti en su versión 8.4.3. A través de este se pudieron relacionar las diferentes categorías y subcategorías subyacentes de la literatura científica. En la figura 2 se muestran las coocurrencias establecidas.

FASE 2. Cada autor, de manera independiente, leyó los trabajos al completo y buscó identificar los siguientes datos previamente consensuados: pertenencia a la categoría principal; subcategoría; tipo de red analizada; nivel educativo; rol educativo del grupo objeto de estudio; contexto (tipo de centro, estudios al que se dirige la investigación, ámbito espacial); metodología empleada; utilización subsidiaria a la TU\&G de otro modelo, teoría o marco; instrumento; hallazgos e implicaciones. Se consideró que la observación de estos datos era fundamental para capturar la esencia de los estudios analizados y poder clasificarlos. Se presenta a continuación la tabla-resumen de los datos (tabla 3) fundamentales de los 39 estudios.

\section{Resultados}

En este epígrafe se resumen los resultados de los trabajos de investigación revisados y clasificados en las tres categorías principales de la codificación temática.

\section{Investigación educativa}

En los trabajos en los que se analizaba el uso que estudiantes y profesores hacían de los espacios comunita- 


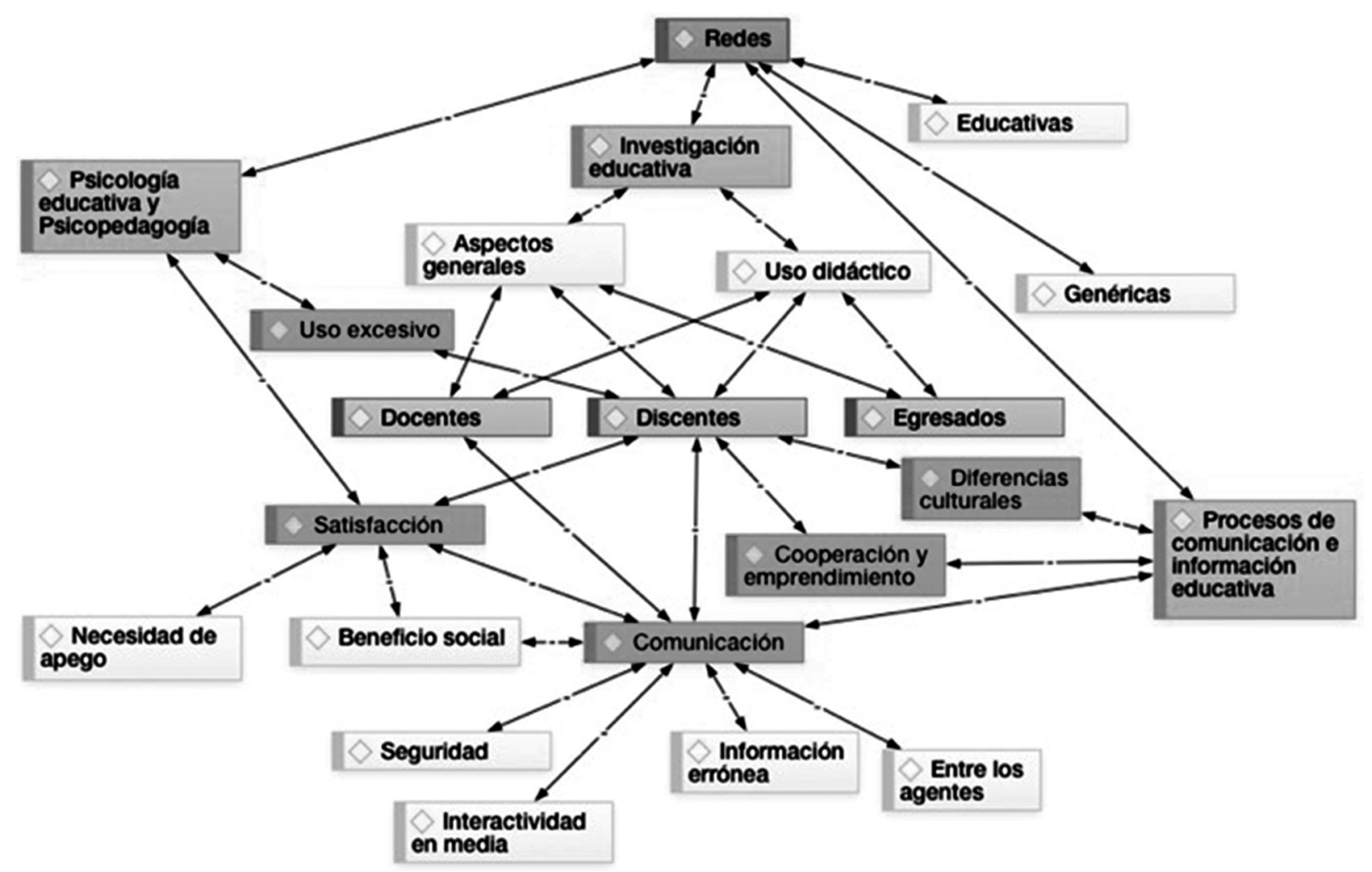

Figura 2. Relación entre los códigos extraídos del análisis cualitativo

Tabla 3. Resumen de los resultados

\begin{tabular}{|c|c|c|c|c|c|c|c|}
\hline & & & 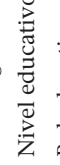 & 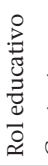 & 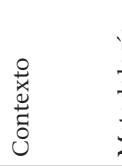 & 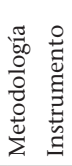 & 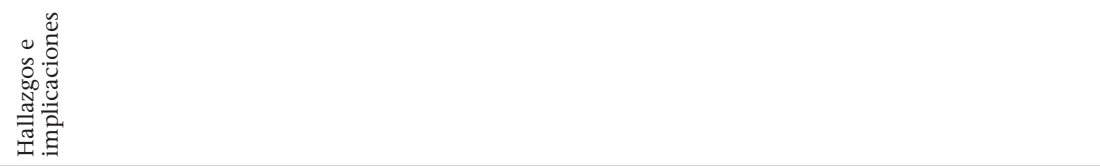 \\
\hline $\begin{array}{l}\text { Vrocharidou y } \\
\text { Efthymiou } \\
\text { (2012) }\end{array}$ & 1 & 6 & 2 & 1 & Grecia & 11 & $\begin{array}{l}\text { Se proporcionan evidencias del uso de la comunicación mediada por computadora y se presentan las } \\
\text { implicaciones para implementar políticas académicas adicionales en ámbitos universitarios. }\end{array}$ \\
\hline $\begin{array}{l}\text { Mingle y } \\
\text { Adams (2015) }\end{array}$ & 1 & 1 & 2 & 1 & Ghana & 32 & $\begin{array}{l}\text { Uso de WhatsApp y Facebook para socializar. Efectos negativos en el rendimiento académico y adicción } \\
\text { a las redes sociales. Sin embargo, algunos experimentaron una mejora de la competencia lectora. Se } \\
\text { recomienda la promoción del uso académico. }\end{array}$ \\
\hline $\begin{array}{l}\text { Yakin y Tinmaz } \\
(2015)\end{array}$ & 1 & 6 & 3 & 3 & $\begin{array}{l}\text { Varios } \\
\text { países }\end{array}$ & 42 & $\begin{array}{l}\text { Combinado con la Teoría de las Redes Sociales. Se ofrecen pautas para que los investigadores y } \\
\text { profesionales integren la TU\&G con otras teorías de manera beneficiosa en futras investigaciones. }\end{array}$ \\
\hline $\begin{array}{l}\text { Dermentzi et } \\
\text { al. (2016) }\end{array}$ & 1 & 6 & 2 & 2 & $\begin{array}{l}\text { Varios } \\
\text { países }\end{array}$ & $1 \quad 1$ & $\begin{array}{l}\text { Combinado con la Teoría Descompuesta del Comportamiento Planificado. Hay necesidad de capacitar } \\
\text { a los académicos en el uso de las redes sociales. Se deben utilizar para la promoción interna. }\end{array}$ \\
\hline $\begin{array}{l}\text { Ahern et al. } \\
\text { (2016) }\end{array}$ & 1 & 1 & 2 & 1 & Irlanda & 12 & $\begin{array}{l}\text { Facebook satisface la necesidad de información y de toma de decisiones. Se consideran implicaciones } \\
\text { para los diseñadores de software basado en las redes. }\end{array}$ \\
\hline $\begin{array}{l}\text { Gallego et al. } \\
\text { (2016) }\end{array}$ & 1 & 1 & 2 & 1 & España & $1 \quad 1$ & $\begin{array}{l}\text { Second Life ofrece una experiencia positiva para el estudiante. Se dan implicaciones académicas, } \\
\text { gerenciales y tecnológicas. }\end{array}$ \\
\hline $\begin{array}{l}\text { Purnawarman } \\
\text { et al. (2016) }\end{array}$ & 1 & 1 & 1 & 1 & Java & 23 & $\begin{array}{l}\text { Se identifican los problemas de uso de Edmodo entre los estudiantes de inglés y se presentan } \\
\text { sugerencias para autoridades e investigadores. }\end{array}$ \\
\hline $\begin{array}{l}\text { Liao et al. } \\
\text { (2016) }\end{array}$ & 1 & 1 & 2 & 1 & Taiwán 3 & 31 & $\begin{array}{l}\text { Combinado con Teoría de la Interacción Social y la Teoría del Compromiso y la Confianza. Los } \\
\text { resultados de este estudio, centrado en Facebook, contribuyen al desarrollo del aprendizaje basado en la } \\
\text { nube y ofrece indicaciones para el diseño de materiales. }\end{array}$ \\
\hline $\begin{array}{l}\text { Choi y Behm- } \\
\text { Morawitz } \\
\text { (2017) }\end{array}$ & 1 & 1 & 2 & 1 & EE. UU. 1 & 13 & $\begin{array}{l}\text { Combinado con STEAM y con teorías cognitivas sociales. Los creadores de contenido de YouTube } \\
\text { pueden educar a los espectadores sobre los usos de la tecnología. Implicaciones significativas para los } \\
\text { enfoques de alfabetización digital. }\end{array}$ \\
\hline $\begin{array}{l}\text { Scholtz et al. } \\
\text { (2017) }\end{array}$ & 1 & 1 & 2 & 1 & Sudáfrica 2 & 23 & $\begin{array}{l}\text { Centrado en Facebook y YouTube. Se encuentra una correlación positiva entre la actividad en una } \\
\text { campaña de redes sociales sobre concienciación ambiental. Se identifican tres factores que influyen: } \\
\text { tiempo, actitud y una conexión rápida a Internet. }\end{array}$ \\
\hline $\begin{array}{l}\text { Durak, G. } \\
(2017)\end{array}$ & 1 & 1 & 2 & 2 & Turquía 3 & 32 & $\begin{array}{l}\text { Combinado con la Teoría de Difusión de Innovación. Se concluye que Edmodo al estar centrada en } \\
\text { educación, no involucra componentes innecesarios. Se recomienda su uso. }\end{array}$ \\
\hline $\begin{array}{l}\text { Ghareb et al. } \\
(2018)\end{array}$ & 1 & 6 & 1,2 & & Kurdistán 1 & 112 & $\begin{array}{l}\text { Las redes de aprendizaje social en la universidad aumentan la orientación académica de los usuarios, } \\
\text { pero falta experiencia y capacitación. }\end{array}$ \\
\hline $\begin{array}{l}\text { Rathnayake y } \\
\text { Winter (2018) }\end{array}$ & 1 & 6 & 2 & 1 & Hawái & 11 & $\begin{array}{l}\text { Combinado con el modelo MAIN. Los estudios futuros deben considerar las diferencias entre } \\
\text { plataformas porque afectan a la gratificación. }\end{array}$ \\
\hline $\begin{array}{l}\text { Thongsri et al. } \\
\text { (2018) }\end{array}$ & 1 & 6 & 2 & 1 & Tailandia 1 & 11 & $\begin{array}{l}\text { Combinado con la Teoría Unificada de Aceptación y Uso de la tecnología. Se detectan factores } \\
\text { favorables para la intención de usar } m \text {-learning y se dan pautas para tal fin. }\end{array}$ \\
\hline $\begin{array}{l}\text { Kim y Malek } \\
(2018)\end{array}$ & 1 & 6 & 2 & 1 & EE. UU. 1 & 11 & $\begin{array}{l}\text { LinkedIn ofrece más beneficios y menos desventajas que Facebook para los estudiantes, y se debe tener } \\
\text { en cuenta para las prácticas docentes y de negocio. }\end{array}$ \\
\hline
\end{tabular}


Tabla 3. Resumen de los resultados (Cont.)

\begin{tabular}{|c|c|c|c|c|c|c|c|}
\hline & 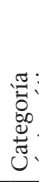 & & 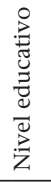 & 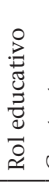 & 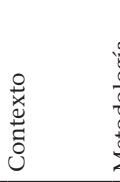 & 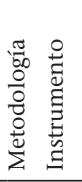 & 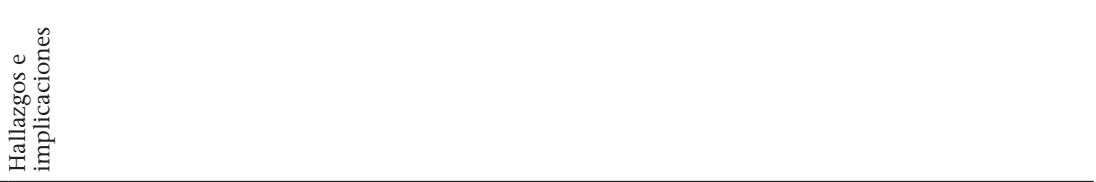 \\
\hline $\begin{array}{l}\text { Gruzd, A. et al. } \\
(2018)\end{array}$ & 1 & 6 & 2 & 2 & $\begin{array}{l}\text { Varios } \\
\text { países }\end{array}$ & 12 & $\begin{array}{l}\text { Se encuentran resultados no solo de los usos, sino también de los propósitos, con implicaciones para la } \\
\text { enseñanza. }\end{array}$ \\
\hline $\begin{array}{l}\text { Saini y } \\
\text { Abraham } \\
(2019)\end{array}$ & 1 & 6 & 2 & 1 & India & 11 & $\begin{array}{l}\text { Construcción de un modelo validado. Se agrega la influencia motivacional como un nuevo factor para } \\
\text { aumentar la capacidad predictiva del modelo de Mazman y Usluel (2010). }\end{array}$ \\
\hline $\begin{array}{l}\text { Goh et al. } \\
(2019)\end{array}$ & 1 & 1 & 2 & 1 & Malasia 1 & 12 & $\begin{array}{l}\text { Combinado con la Teoría de la Cadena Final de Medios. Se obtuvieron hallazgos sobre los } \\
\text { determinantes y el efecto del uso de Facebook sobre el rendimiento académico y la comunicación entre } \\
\text { estudiantes }\end{array}$ \\
\hline $\begin{array}{l}\text { Yen et al. } \\
(2019)\end{array}$ & 1 & 1 & 1 & 1 & Taiwán & 11 & $\begin{array}{l}\text { Centrado en YouTube. Se considera que el entretenimiento, la conveniencia y la información son las } \\
\text { principales gratificaciones que se obtienen al aprender de los vídeos de las celebridades. }\end{array}$ \\
\hline $\begin{array}{l}\text { Cozma y Hallaq } \\
(2019)\end{array}$ & 1 & 1 & 2 & 1 & EE. UU. 3 & 33 & $\begin{array}{l}\text { Centrado en Twitter. Se sugiere que la educación en periodismo está rezagada con respecto a los } \\
\text { cambios en la industria. }\end{array}$ \\
\hline $\begin{array}{l}\text { Dalvi-Esfahani } \\
\text { et al. }(2020)\end{array}$ & 1 & 6 & 2 & 1 & Malasia 1 & 11 & $\begin{array}{l}\text { Combinado con el Modelo de Aceptación de Tecnología y la Teoría del Comportamiento Planificado. El } \\
\text { aprendizaje en red percibido se explica significativamente por su intención conductual y se debe seguir } \\
\text { investigando. }\end{array}$ \\
\hline $\begin{array}{l}\text { Cozma y } \\
\text { Dimitrova } \\
(2020)\end{array}$ & 1 & 1 & 2 & 2 & EE. UU. 2 & 22 & $\begin{array}{l}\text { Los académicos motivados extrínsecamente por la investigación actualizan sus cuentas de ResearchGate } \\
\text { con más frecuencia, pero obtienen menos beneficios de la plataforma. }\end{array}$ \\
\hline $\begin{array}{l}\text { Özad y Uyga } \\
(2014)\end{array}$ & 2 & 5 & 2 & 1 & Chipre & 11 & $\begin{array}{l}\text { Las redes sociales juegan un papel importante en la satisfacción de la necesidad de apego entre los } \\
\text { jóvenes que se encuentran en el inicio de su educación terciaria. }\end{array}$ \\
\hline $\begin{array}{l}\text { Dhir et al. } \\
(2017)\end{array}$ & 2 & 3 & 1 & 1 & India & 12 & $\begin{array}{l}\text { El uso intensivo de Facebook se puede predecir mediante la TU\&G. Se ofrecen sugerencias para } \\
\text { educadores, expertos en pedagogía y responsables de políticas educativas. }\end{array}$ \\
\hline $\begin{array}{l}\text { Islam et al. } \\
(2018)\end{array}$ & 2 & 3 & 2 & 1 & Pakistán 1 & 11 & $\begin{array}{l}\text { El entretenimiento, la interacción social, la búsqueda de información y los motivos económicos juegan } \\
\text { un papel significativo en el uso excesivo de Internet y esto impacta negativamente en el rendimiento } \\
\text { académico. }\end{array}$ \\
\hline $\begin{array}{l}\text { Klobas et al. } \\
(2018)\end{array}$ & 2 & 3 & 2 & 1 & Malasia 1 & 12 & $\begin{array}{l}\text { Los educadores deben tener en cuenta los riesgos del uso compulsivo de YouTube cuando diseñan } \\
\text { recursos para sus estudiantes. Los riesgos se minimizan alertando a los alumnos y ayudándolos a su } \\
\text { autodesarrollo con estrategias de manejo. }\end{array}$ \\
\hline $\begin{array}{l}\text { Raza et al. } \\
(2020)\end{array}$ & 2 & 5 & 2 & 1 & Pakistán 1 & 11 & $\begin{array}{l}\text { Se detectan las motivaciones de uso de las redes sociales por parte de los estudiantes. Las instituciones } \\
\text { educativas pueden utilizar los resultados de la investigación incorporando herramientas de las redes } \\
\text { sociales en el sistema académico. }\end{array}$ \\
\hline Aduloju (2020) & 2 & 3 & 2 & 1 & Nigeria & 12 & $\begin{array}{l}\text { Los estudiantes de pregrado usan las redes sociales principalmente para socializar y solo con fines } \\
\text { académicos cuando se les asigna tareas o cuando investigan. Se recomienda la educación en } \\
\text { alfabetización mediática. }\end{array}$ \\
\hline $\begin{array}{l}\text { Zhou et al. } \\
\text { (2011) }\end{array}$ & 3 & 2 & 3 & 3 & $\begin{array}{l}\text { Varios } \\
\text { países }\end{array}$ & 12 & $\begin{array}{l}\text { El uso de Second Life se debe a tres motivaciones: funcional, experiencial y social. Los usuarios } \\
\text { experimentados son más conscientes de los valores de esta red para la creación, la educación y el } \\
\text { comercio. }\end{array}$ \\
\hline $\begin{array}{l}\text { Hossain y } \\
\text { Veenstra (2013) }\end{array}$ & 3 & 2 & 2 & 1 & EE. UU. 1 & 12 & $\begin{array}{l}\text { Los desplazamientos geográficos y físicos no siempre influyen en que los estudiantes mantengan } \\
\text { relaciones a través de las redes sociales. }\end{array}$ \\
\hline $\begin{array}{l}\text { Chen et al. } \\
\text { (2015) }\end{array}$ & 3 & 4 & 3 & 1 & Singapur 1 & 12 & $\begin{array}{l}\text { La capacitación en alfabetización informacional debería abordar las motivaciones sociales que la } \\
\text { impulsan. Las redes sociales pueden desarrollar características que permitan detectar la información } \\
\text { errónea. }\end{array}$ \\
\hline $\begin{array}{l}\text { Sarapin y } \\
\text { Morris (2015) }\end{array}$ & 3 & 4 & 2 & 2 & EE. UU. 1 & 12 & $\begin{array}{l}\text { La participación de la facultad en la interacción no académica a través de Facebook es muy idónea para } \\
\text { aumentar la percepción de los estudiantes sobre su experiencia universitaria y su desempeño } \\
\text { académico. }\end{array}$ \\
\hline $\begin{array}{l}\text { Zhang y Rau } \\
\text { (2015) }\end{array}$ & 3 & 4 & 2 & 1 & China & 23 & $\begin{array}{l}\text { La multitarea con contenido relevante en diferentes dispositivos satisface la necesidad cognitiva, pero } \\
\text { la que es con contenido irrelevante sirve para satisfacer la necesidad emocional. Se dan indicaciones } \\
\text { para diseñar experiencias de usuario. }\end{array}$ \\
\hline Yen (2016) & 3 & 4 & 2 & 2 & Taiwán & 12 & $\begin{array}{l}\text { Combinado con la Teoría del Capital Social y la Teoría de la Identidad Social. El capital y la identidad } \\
\text { social tienen un impacto en la intención de compartir conocimientos de los docentes en Facebook. }\end{array}$ \\
\hline $\begin{array}{l}\text { Sheldon et al. } \\
\text { (2017) }\end{array}$ & 3 & 2 & 2 & 1 & $\begin{array}{l}\text { Croacia y } 1 \\
\text { EE. UU. }\end{array}$ & 12 & $\begin{array}{l}\text { El uso de Instagram por parte de los estudiantes croatas refleja tendencias colectivistas, principalmente } \\
\text { interacción social, mientras que el de los estudiantes estadounidenses refleja tendencias individualistas, } \\
\text { principalmente, la autopromoción y la documentación. }\end{array}$ \\
\hline Ma et al. (2019) & 3 & 4 & 2 & 1 & China & 13 & La seguridad del usuario influye en una mayor adquisición de las motivaciones de uso. \\
\hline $\begin{array}{l}\text { Wu y Song } \\
(2019)\end{array}$ & 3 & 4 & 3 & 1 & Taiwán & 12 & $\begin{array}{l}\text { Los asistentes a cursos para el emprendimiento en línea valoran la confianza como principal factor de } \\
\text { gratificación. }\end{array}$ \\
\hline $\begin{array}{l}\text { Perera et al. } \\
(2020)\end{array}$ & 3 & 4 & 2 & 1 & Sri Lanka 1 & 11 & $\begin{array}{l}\text { El compromiso social de los estudiantes con las universidades a través de las redes sociales desarrolla un } \\
\text { posicionamiento de marca distintivo de dichas universidades entre los estudiantes. }\end{array}$ \\
\hline \multicolumn{8}{|c|}{$\begin{array}{l}\text { Categoría temática: } 1 \text { = investigación educativa; } 2=\text { psicología educativa y psicopedagogía; } \\
\text { Subcategoría: } 1 \text { = uso didáctico; } 2=\text { diferencias culturales; } 3 \text { = uso excesivo; } 4 \text { = comunicaci } \\
\text { Nivel educativo: } 1=\text { secundaria; } 2=\text { universidad; } 3=\text { genérico } \\
\text { Rol educativo: } 1=\text { alumnado; } 2 \text { docentes; } 3=\text { genérico } \\
\text { Metodología: } 1 \text { = cuantitativa; } 2=\text { cualitativa; } 3=\text { mixta; } 4 \text { = revisión sistemática o teórica } \\
\text { Instrumento: } 1 \text { = adaptado; } 2=\text { ad hoc; } 3=\text { estudio de caso }\end{array}$} \\
\hline
\end{tabular}

rios mediados por las redes sociales desde la perspectiva de la TU\&G, se pudo distinguir entre los que abordaban aspectos genéricos y los que aplicaban el estudio al uso de una red específica.

De los trabajos analizados ninguno realizaba revisiones sistemáticas. Sin embargo, hubo un caso que abordaba el diseño de estudios de redes sociales con propósitos de instrucción y los atributos comunes de las teorías y áreas de aplicación específicas desde una perspectiva teórica. Se trata del trabajo de Yakin y Tinmaz (2015) que, basándose en la TU\&G, proporcionaba una guía teórica para el uso educativo de las redes sociales. Estos autores aplicaron de manera conjunta la TU\&G con la Teoría de las Redes Sociales, y atendieron a los principios del conectivismo y el constructivismo para ofrecer pautas de diseño.

De entre los artículos que contemplaban perspectivas más generales de las redes, el más distante en el 
tiempo de esta categoría fue el de Vrocharidou y Efthymiou (2012). Sus autores estudiaron las motivaciones de uso del mundo digital -redes, correo y mensajería de manera transversal- entre estudiantes universitarios para predecir su frecuencia y sostenibilidad. Concluyeron que el uso estaba más correlacionado con las necesidades que con la experiencia previa, y que era necesario implementar políticas académicas en ese sentido. Atendiendo al desempeño del profesorado y de los investigadores, Dermentzi et al. (2016) combinaron la Teoría Descompuesta del Comportamiento Planificado con la TU\&G para comparar el uso de las redes sociales con el de otras tecnologías digitales entre los académicos. El resultado fue que las redes sociales eran más adecuadas para establecer comunidades de trabajo, mientras que el resto de los medios tecnológicos resultaban más útiles para buscar información y adoptar una determinada identidad e imagen profesional pública. Recomendaron que las universidades intensificasen los medios de promoción interna. Ghareb et al. (2018) compararon el uso de redes sociales genéricas y redes sociales en estudiantes y profesores de educación superior. Consideraron que las genéricas carecían de determinadas utilidades y que, a veces, alojaban contenidos inapropiados para la educación. Kim y Malek (2018) establecieron la misma comparación entre el uso de las redes genéricas y las redes profesionales por parte de los estudiantes universitarios, y consideraron que Facebook tenía muchas desventajas y carencias frente a los beneficios percibidos en LinkedIn. Es interesante tomar en cuenta aspectos particulares de estos cuatro estudios, pues presentaban las mismas recomendaciones a raíz de sus hallazgos -aun ocupándose de contextos diferentes y estar distanciados en el tiempo-. Las conclusiones y recomendaciones fueron muy similares en las poblaciones analizadas: alumnos de la escuela de negocios (Vrocharidou \& Efthymiou, 2012), académicos de diferentes universidades (Dermentzi et al., 2016), académicos de Kurdistán (Ghareb et al., 2018), o estudiantes de hostelería (Kim \& Malek, 2018). En todos los ámbitos se aconsejaba intensificar el uso de las redes sociales tras aplicar la TU\&G, y más concretamente, en los dos últimos, preferiblemente utilizando redes académicas o profesionales.

Rathnayake y Winter (2018) conceptualizaron la TU\&G a través del modelo MAIN (Modality, Agency, Interactivity, and Navigability) y pusieron de manifiesto la necesidad de distinguir entre las diferentes plataformas. También Thongsri et al. (2018) combinaron la TU\&G de motivación con una teoría de uso de tecnología, la Teoría Unificada de Aceptación y Uso de la Tecnología, y pusieron de manifiesto que las necesidades cognitivas, afectivas y sociales tuvieron un efecto significativo sobre la intención de utilizar M-Learning y demostraron la conveniencia del uso de dispositivos móviles para gestionar las redes sociales educativas. Dalvi-Esfahani et al. (2020) también se ocuparon de las redes mediadas por dispositivos móviles y exploraron el aprendizaje percibido por los estudiantes; detectaron los factores de gratificación y discutieron las implicaciones prácticas. Ambos trabajos (Thongsri et al., 2018; Dalvi-Esfahani et al., 2020) combinaron la TU\&G con la Teoría y Uso de la Tecnología para ocuparse de un vehículo relevante en el uso de las redes sociales: el teléfono móvil. Los autores emplearon metodologías similares, utilizando para su investigación cuestionarios ad hoc basados en la combinación de ambas teorías y en un contexto de alumnos universitarios de diferentes disciplinas; y los dos trabajos pusieron el acento en la intención de uso -que era positiva en los dos casos- y concluyeron que se debía seguir investigando para ofrecer códigos de buenas prácticas. Cabe resaltar que fueron escasos los estudios que detectaban los usos y las gratificaciones de las redes mediados por dispositivos móviles en el ámbito educativo, lo que abre una perspectiva para la investigación tan necesaria como interesante.

Gruzd et al. (2018) utilizaron el marco de la TU\&G para buscar factores de uso y necesidades docentes entre el profesorado universitario, y descubrieron una nueva gama de propósitos. Saini y Abraham (2019) realizaron un exhaustivo estudio, que puede considerarse paradigmático desde el punto de vista de la metodología, pues utilizaron de manera integrada la TU\&G y el modelo estructural propuesto por Mazman y Usluel (2010) e incluyeron la influencia motivacional como constructo adicional. Evaluaron las percepciones de futuros docentes aplicando los principios de la TU\&G y abrieron el campo de estudio a este nuevo constructo que aunó ambos parámetros. Consiguieron explicar el $69.1 \%$ de la varianza en el uso de las diferentes redes sociales genéricas y aportaron distintas implicaciones prácticas. Los trabajos más numerosos fueron los que atendían al uso de determinadas redes, ya fueran educativas, profesionales o genéricas. Por ejemplo, Purnawarman et al. (2016) y Durak (2017) se centraron en el uso de una red social específica para el mundo educativo: Edmodo. Los primeros utilizaron un enfoque de estudio de caso con una perspectiva de género y se estudiaron las limitaciones manifestadas por los estudiantes. Presentaron sugerencias de cara a implementar políticas educativas e iniciar nuevas áreas de investigación. Durak (2017) también consideró el liderazgo de uso en esta red y combinó la TU\&G con la de Teoría de Difusión e Innovación. Comparó Edmodo con las redes genéricas y destacó los beneficios de la primera al considerar que descartaba componentes innecesarios. En este aspecto, cabe reseñar que la mayor parte de los trabajos analizados y que contemplaban tanto redes genéricas como profesionales o académicas, llegaban a la misma conclusión: las redes específicas eliminaban distracciones y otros factores negativos, como la falta de privacidad.

Ahern et al. (2016), Liao et al. (2016) y Goh et al. (2019) estudiaron el uso de Facebook desde la perspectiva de TU\&G. Los primeros analizaron los motivos que llevaban a los estudiantes a utilizar grupos de Facebook, y concluyeron que se debía al hecho de que satisfacían su necesidad de información y toma de decisiones y 
daban ideas para su implementación. Liao et al. (2016) establecieron los parámetros para diseñar una plataforma en línea que integrara la configuración de los e-books con las funciones sociales de Facebook e investigaron el comportamiento de aprendizaje de los estudiantes. Goh et al. (2019) examinaron la misma red para determinar el logro académico percibido entre los estudiantes y concluyeron que afectaba positivamente al rendimiento académico. Por lo tanto, se puede determinar que los autores que analizaron las redes genéricas y específicas de manera conjunta prefirieron las segundas para las prácticas académicas. No obstante, la utilidad de las redes sociales genéricas en el ámbito educativo también es innegable, como muestran los datos presentados en estos trabajos -en concreto el de Liao et al. (2016) o el de Goh et al. (2019)- en los que, a pesar de detectar limitaciones, ofrecían recomendaciones para implementar el efecto académico positivo de una red general concreta, como es el caso de Facebook.

Mingle y Adams (2015) analizaron el uso de las redes WhatsApp y Facebook entre estudiantes de secundaria para determinar cómo implementar sus efectos académicos positivos en disciplinas como la ortografía, la gramática y el desempeño académico en general. Concluyeron que estas redes se usaban generalmente con fines sociales, y que los efectos producidos eran nocivos dado el tiempo que invertían los estudiantes, si bien algunos mejoraron en habilidades lectoras y en la capacidad de debatir y compartir. Hicieron recomendaciones para que la política educativa limitara los efectos adversos. Es de reseñar que así bien la mayoría de los trabajos se centraba en los beneficios de las redes sociales en el ámbito educativo y en cómo implementarlos, resultan muy interesantes -y en especial para el nivel educativo de secundaria- los que, como en este caso, ofrecen una prospectiva clara para superar las limitaciones o los posibles problemas, ya que proporcionan indicaciones sobre cómo abordarlos $\mathrm{y}$, por lo tanto, pueden ser útiles para los docentes.

Choi y Behm-Morawitz (2017), y Yen et al. (2019) se ocuparon de los beneficios educativos de los vídeos producidos por youtubers y celebridades. Los primeros examinaron contenidos relacionados con la belleza, el maquillaje y la moda como medio de alfabetización digital y aplicación del enfoque STEAM; consideraron que estos vídeos motivaban a los consumidores a aprender a producir contenidos. Los segundos, Yen (2016) et al. (2019) estudiaron el uso de vídeos de celebridades para aprender sobre finanzas empresariales y concluyeron que el entretenimiento, la comodidad y la información eran las principales gratificaciones que se obtenían al aprender de estos vídeos.

Gallego et al. (2016) analizaron la motivación de uso de Second Life en cursos de aprendizaje en línea y extrajeron conclusiones e implicaciones académicas, gerenciales y tecnológicas. Cozma y Hallaq (2019) analizaron el uso de Twitter entre estudiantes de periodismo como medio de promoción de los seguidores de sus canales de televisión en sus campus y llegaron a la conclusión de que debían mejorar sus prácticas en esta red social para obtener mayores beneficios. Cozma y Dimitrova (2020) se ocuparon del uso de la red académica ResearchGate y llegaron a determinar que los académicos motivados extrínsecamente por la investigación actualizaban sus cuentas con más frecuencia, sin embargo, obtenían menos beneficios de la plataforma; este hallazgo puede considerarse de especial relevancia para los académicos e investigadores por la reflexión que conlleva y sus implicaciones prácticas para el desempeño de las relaciones entre pares en el ámbito universitario.

En este ámbito existe un trabajo dedicado a la evaluación del uso de las redes sociales entre universitarios en el ámbito extracurricular. Scholtz et al. (2017) utilizaron como tema del estudio de caso la concienciación medioambiental y se usaron tres gratificaciones de la TU\&G: coordinación, acceso inmediato y presencia social; se encontró una correlación positiva entre la actividad de la campaña en las redes sociales y el conocimiento ambiental, pero también se detectó que la aceptación de las redes sociales utilizadas con fines educativos extracurriculares eran bajas y, por lo tanto, se deberían poner los medios para evitar esta realidad. Resulta interesante la investigación aplicando la TU\&G a las redes sociales en contextos no formales, pues es otro aspecto poco trabajado y que abre un campo de indagación amplio y fructífero.

\section{Psicología educativa}

Los trabajos que adoptaron la perspectiva psicológica se ocuparon de buscar beneficios personales en el uso de las mismas o en detectar el empleo excesivo de las redes entre los alumnos y buscar la explicación de este hecho a través de la TU\&G para poder limitar esta situación.

Dos trabajos se focalizaban en la búsqueda de aspectos satisfactorios en las redes. Özad y Uygarer (2014) se ocuparon de las redes como respuesta a las necesidades afectivas de los estudiantes y su papel como conformadoras de relaciones personales. Profundizaron en el contraste entre las conexiones en el mundo real y en las redes, y concluyeron que las redes tenían un papel relevante para satisfacer necesidades de apego. Raza et al. (2020) examinaron el papel de las redes en relación con la satisfacción social de los estudiantes enfocándose en los resultados psicológicos y sus resultados mostraron la mejora de la vida de los estudiantes al obtener beneficios sociales, si bien detectaron el peligro de la "sobrecarga social".

Varios trabajos que adoptaban la perspectiva psicológica se ocuparon del uso excesivo de las redes entre los alumnos, y buscaron la explicación de este hecho a través de la teoría TU\&G para poder limitar esta situación. Dhir et al. (2017) investigaron sobre un debate que nació a la vez que el propio uso educativo de las redes, en este caso aplicado a Facebook: por un lado, existía la recomendación de utilizarlas en el aula, por otro lado, la desconfianza de los docentes y la creencia de que un uso demasiado intensivo pudiera tener efec- 
tos negativos sobre el aprendizaje; esta brecha se abordó a través de la TU\&G, y se concluyó que su aplicación no predecía un exceso de uso significativo en el aula y desarrollaron implicaciones prácticas para educadores y pedagogos.

Islam et al. (2018a, 2018b), en sus dos artículos, también se ocuparon del uso intensivo de Internet, y entre otros medios, de las redes en el ámbito universitario; los resultados indicaron que factores, como el entretenimiento, los motivos económicos y la interacción social, generaban un uso excesivo y provocaban un impacto negativo. Klobas et al. (2018) analizaron el abuso de YouTube entre los estudiantes universitarios, y compararon la motivación de conseguir información académica con la de entretenimiento, esta última se asociaba al uso compulsivo, y de este modo la motivación informativa quedaba en un segundo plano. Se tuvieron en cuenta los rasgos de personalidad de los estudiantes, pero se concluyó que los efectos de motivación eran independientes de la personalidad. Aduloju (2020) trabajó la misma dicotomía en el ámbito espacial de Nigeria y se focalizó en analizar el tiempo y grado de motivación que empleaban los estudiantes en el uso académico de las redes. De este modo, al igual que en otros trabajos anteriormente descritos, demostraron que los estudiantes empleaban más tiempo en las redes genéricas para socializar que para fines académicos y que el tiempo invertido en las redes redundaba en resultados negativos, por lo que convendría implementar experiencias de alfabetización mediática.

Por lo tanto, los trabajos que abordaban los usos y las gratificaciones en las redes sociales desde la perspectiva de la psicología educativa -como sucedía con los que pertenecían a la investigación educativa- ponían el acento en la "sobrecarga" social y el exceso de tiempo invertido, entre otros efectos adversos, pero ofrecían una visión positiva al señalar cómo atajar estos problemas. Al aplicar la TU\&G en sus estudios, los altos índices de motivación y de intención de uso revelaban la necesidad de seguir utilizando las redes a pesar de sus posibles inconvenientes.

\section{Comunicación e información educativa}

Las investigaciones que usaban la TU\&G más apegadas a aspectos comunicativos e informacionales se ocuparon de perspectivas etnográficas, demográficas, sociales y culturales, temas relacionados con el emprendimiento y la iniciativa, y formas de comunicación entre los actores. Todos estos temas son tratados desde la perspectiva de la seguridad y la información veraz.

En cuanto a los usos diferenciales de las redes, Zhou et al. (2011) estudiaron los factores que atraían a distintos colectivos hacia el uso de redes, en este caso Second Life; los usuarios de género femenino, de mayor edad y con mayor nivel educativo de educación se mostraron más proclives a otorgar a la plataforma un uso pedagógico educativo o investigador. Hossain y Veenstra (2013) compararon el uso de las redes por parte de los estudiantes autóctonos y foráneos de Es- tados Unidos, y concluyeron que los desplazamientos no siempre eran un factor para mantener relaciones a través de las redes. Sheldon et al. (2017) compararon el uso de Instagram que realizaban los alumnos universitarios de una cultura individualista, como Estados Unidos, con el que se hacía en Croacia, donde existía una cultura colectivista; las motivaciones no variaron, pero sí aspectos como las gratificaciones, los usos y la autopromoción.

En cuanto a las prácticas de comunicación, acción colectiva y emprendimiento, Yen (2016) investigó la intención de "compartir conocimiento" entre los usuarios de las redes en el mundo educativo; y los resultados mostraron que el capital social y la identidad tenían un impacto positivo en la intención de compartir conocimientos entre los docentes, lo que influía en la intención de adhesión colectiva a las redes. Wu y Song (2019) exploraron los usos de las redes y las gratificaciones entre personas que cursaban estudios no universitarios de emprendimiento; los factores variaron en relación con las tres redes sociales estudiadas y el factor confianza fue un aspecto que cabía destacar entre los emprendedores en línea. Perera et al. (2020) investigaron la influencia que ejercía el compromiso establecido por los estudiantes en las redes en relación a la marca social de las instituciones universitarias y concluyeron que cuando dicho compromiso era alto, se desarrollaba un posicionamiento de marca distintivo entre las instituciones.

En relación con los procesos de comunicación mediados por redes entre los distintos agentes del ámbito educativo, Sarapin y Morris (2015) indagaron sobre las relaciones entre alumnos y profesores en el ámbito universitario; para ello, estudiaron las expectativas de los docentes en cuanto a las percepciones de sus alumnos sobre aspectos como la credibilidad, la profesionalidad o la accesibilidad. Compararon los resultados con la escala de compensación esperada por los profesores y llegaron a la conclusión de que la interacción no académica a través de las redes mejoraba la experiencia universitaria y el desempeño académico de los alumnos. Zhang y Rau (2015) estudiaron la interacción entre múltiples tareas mediadas por dispositivos inteligentes con la red WeChat. Intentaron detectar sus motivaciones y sentimientos; establecieron relaciones entre las multitareas más y menos relevantes y la satisfacción de necesidades emocionales, y dedujeron que las más irrelevantes producían mayor satisfacción.

Chen et al. (2015) investigaron sobre la necesidad de alfabetización digital entre los estudiantes para poder ser creadores y comunicadores de información eficaces; para ello, indagaron sobre las causas de la diseminación de información errónea y los rumores en las redes sociales. Comprobaron que la mayoría de los factores eran de naturaleza social, y los autores sugirieron desarrollar características, usos y aplicaciones en las redes que animaran a los usuarios a remarcar la información errónea. Ma et al. (2019) estudiaron la educación en seguridad en el ámbito de las redes sociales para indagar sobre su impacto educativo. Sus 
hallazgos demostraron que la conciencia de tener seguridad moderaba las expectativas de entretenimiento de los usuarios y mejoraba la calidad y la solidez de los contenidos.

\section{Reflexión final y conclusiones}

Tras la revisión de los principales contenidos expuestos en la literatura científica en relación con la cuestión abordada, se realizan a continuación diversas reflexiones finales con el objetivo de sintetizar las ideas principales de las contribuciones presentadas.

La aplicación de una teoría destinada desde su origen a detectar las motivaciones de los usuarios actúa como un vehículo eficaz para dirigir y concretar debates sobre el uso de las redes sociales en el ámbito educativo y la mejora de las prácticas docentes.

En este sentido, se puede detectar que la aplicación de la TU\&G otorga una perspectiva muy crítica a los trabajos que la emplean. Sus resultados exponen de manera frecuente las limitaciones detectadas y las prospectivas que hay que tener en cuenta para solventarlas. Asimismo, se ponen en tela de juicio aspectos tales como la distracción que supone el uso de las redes, la escasa inclinación a compartir información académica, los problemas que en ocasiones existen a la hora de configurar comunidades de aprendizaje, o en qué escenarios formativos conviene utilizar las redes genéricas o las específicas. Los estudios reflejan, en unas ocasiones falta de motivación; en otras, escasez de competencia digital; a veces, exigua preparación a la hora de gestionar la identidad profesional; y, en general, una necesidad imperiosa de buscar soluciones para implementar de manera óptima el uso de las redes sociales en educación. La literatura analizada proporciona claves de buenas prácticas para diseñar experiencias y poder tomar de decisiones inclusivas en el aula y, a un nivel más amplio, pretende orientar políticas educativas.

Por todo ello, la TU\&G, aunque hasta ahora se ha aplicado discretamente, se revela como un excelente elemento de diagnosis que muestra su validez como punto de partida para obtener patrones de comportamiento modelados por las dimensiones y los factores que se analizan. La literatura muestra que los resultados que se obtienen al aplicar la TU\&G son eficaces para establecer procesos de investigación-acción, y atender requerimientos sobre competencias digitales en el mundo educativo.

Declaración de divulgación del autor: Los autores declaran no tener conflicto de intereses.

Financiación: Esta investigación se ha realizado en el marco del proyecto RedEDUNIR. El uso de las redes sociales genéricas en el ámbito docente: retos formativos para el contexto educativo actual. (Universidad Internacional de la Rioja)

\section{Referencias}

Aduloju, E.T. (2020). Undergraduate students and time spent on social networking sites: A study of the uni- versity of Port Harcourt, Nigeria. World of Media, 2020 (3), 57-72. https://doi.org/10.30547/worldofmedia.3.2020.3

Ahern, L., Feller, J., \& Nagle, T. (2016). Social media as a support for learning in universities: An empirical study of Facebook groups. Journal of Decision Systems, 25, 3549. https://doi.org/10.1080/12460125.2016.11874

Alexander, P.A. (2020). Methodological guidance paper: The art and science of quality systematic reviews. Review of Educational Research, 90(1), 6-23. https:// doi.org/10.3102/0034654319854352

Chen, C.P. (2018). Understanding mobile Englishlearning gaming adopters in the self-learning market: The Uses and Gratification Expectancy Model. Computers \& Education, 126, 217-230. https://doi. org/10.1016/j.compedu.2018.07.015

Chen, X., Sin, S.C. J., Theng, Y.L., \& Lee, C.S. (2015). Why students share misinformation on social media: Motivation, gender, and study-level differences. The journal of academic librarianship, 41(5), 583-592. https://doi.org/10.1016/j.acalib.2015.07.003

Choi, G.Y., \& Behm-Morawitz, E. (2017). Giving a new makeover to STEAM: Establishing YouTube beauty gurus as digital literacy educators through messages and effects on viewers. Computers in Human Behavior, 73, 80-91. https://doi.org/10.1016/j.chb.2017.03.034

Cozma, R., \& Hallaq, T. (2019). Digital natives as budding journalists: College TV stations' uses of twitter. Journalism and Mass Communication Educator, 74(3), 306317. https://doi.org/10.1177/1077695818805899

Cozma, R., \& Dimitrova, D. (2020). Research gate or revolving door? uses and gratifications of academic social media among communication scholars. Journalism and Mass Communication Educator. October, 2020. https://doi.org/10.1177/1077695820965030

Dalvi-Esfahani, M., Wai Leong, L., Ibrahim, O., \& Nilashi, M. (2020). Explaining students' continuance intention to use mobile web 2.0 learning and their perceived learning: An integrated approach. Journal of Educational Computing Research, 57(8), 1956-2005. https://doi.org/10.1177/0735633118805211

Davis, F.D. (1986). A Technology Acceptance Model for Empirically Testing New End-User Information Systems: Theory and Results. Unpublished doctoral dissertation. Massachusetts Institute of Technology.

Davis, F.D. (1989). Perceived usefulness, perceived ease of use, and user acceptance. MIS Quarterly, 13(3), 319-340.

Dermentzi, E., Papagiannidis, S., Osorio-Toro, C., \& Yannopoulou, N. (2016). Academic engagement: Differences between intention to adopt social networking sites and other online technologies. Computers in Human Behavior, 61, 321-332. https://doi. org/10.1016/j.chb.2016.03.019

Dhir, A., Khalil, A., Lonka, K., \& Tsai, C. C. (2017). Do educational affordances and gratifications drive intensive Facebook use among adolescents? Computers in Human Behavior, 68, 40-50. hhttp://dx.doi. org/10.1016/j.chb.2016.11.0140

Durak, G. (2017). Using Social Learning Networks 
(SLNs) in Higher Education: Edmodo Through the Lenses of Academics. The International Review of Research in Open and Distributed Learning, 18(1). https:// doi.org/10.19173/irrodl.v18i1.2623

Gallego M.D., Bueno S., \& Noyes J. (2016). Second Life adoption in education: A motivational model based on Uses and Gratifications theory, Computers \& Education, 100, 81-93 https://doi.org/ 10.1016/j.compedu.2016.05.001

Ghareb, M.I., Ahmed, Z.A., \& Ameen, A.A. (2018). The role of learning through social network in higher education in KRG. International Journal of Scientific and Technology Research, 7(5), 20-27.

Goh, C., Rasli, A., Tan, O.K., \& Choi, S.L. (2019). Determinants and academic achievement effect of Facebook use in educational communication among university students. Aslib Journal Information Management., 71, 105-123. https://doi.org/10.1108/AJIM05-2018-0116

Gruzd, A., Haythornthwaite, C., Paulin, D., Gilbert, S., \& del Valle, M.E. (2018). Uses and gratifications factors for social media use in teaching: Instructors' perspectives. New Media and Society, 20(2), 475-494. https://doi.org/10.1177/1461444816662933

Hernández-González, V., Reverter-Masia, J. \& JovéDeltell, M.C. (2017). Producción científica de los profesores del área de Educación Física y deportiva en Cataluña (quinquenio 2005-2009 versus 20102014). SPORT TK-Revista EuroAmericana de Ciencias del Deporte, 6(1), 17-24.

Hossain, M.D., \& Veenstra, A.S. (2013). Online maintenance of life domains: Uses of social network sites during graduate education among the US and international students. Computers in Human Behavior, 29(6), 2697-2702. https://doi.org/10.1016/j. chb.2013.07.007

Hsu, M.H., Tien, S.W., Lin, H.C. \& Chang, C.M. (2015) Understanding the roles of cultural differences and socio-economic status in social media continuance intention, Information Technology \& People, 28 (1), 224-241, https://doi.org/10.1108/ITP-01-2014-0007

Hutton, B., Catalá-López, F. \& Moher, D. (2016). La extensión de la declaración PRISMA para revisiones sistemáticas que incorporan metaanálisis en red: PRISMA-NMA. Medicina Clínica, 147 (6), 262-266. https://doi.org/10.1016/j.medcli.2016.02.025

Islam, S., Malik, M. I., Hussain, S., Thursamy, R., Shujahat, M., \& Sajjad, M. (2018a). Motives of excessive Internet use and its impact on the academic performance of business students in Pakistan. Journal of Substance Use, 23(1), 103-111. https://www.tandfonline.com/doi/abs/10.1080/14659891.2017.1358305 ?journalCode=ijsu20

Islam, S., Malik, M. I., Hussain, S., Thursamy, R., Shujahat, M., \& Sajjad, M. (2018b). Motives of excessive Internet use and its impact on the academic performance of business students in Pakistan. Journal of Substance Use, 23(3), 254-261. https://doi.org/10.10 80/14659891.2017.1388857

Katz, E. (1959). Mass communication research and the study of culture. Studies in Public Communication, 2, $1-6$.

Katz, E., Blumler, J.G., \& Gurevitch, M. (1974). Utilization of mass communication by the individual. In J. G. Blumler \& E. Katz (Eds.), The Use of Mass Communications: Current Perspectives on Gratifications Research (pp. 19-32). Beverly Hills, CA

Kim, W., \& Malek, K. (2018). Social networking sites versus professional networking sites: Perceptions of hospitality students. Journal of Human Resources in Hospitality and Tourism, 17(2), 200-221. https://doi. org/10.1080/15332845.2017.1340763

Klobas J.E., McGill T.J., Moghavvemi S. \& Paramanathan T., (2018). Compulsive YouTube usage: A comparison of use motivation and personality effects, Computers in Human Behavior, 86, 129-139, https:// doi.org/10.1016/j.chb.2018.05.038

Lee, C.S. \& Ma, L. (2012), News sharing in social media: the effect of gratifications and prior experience, Computers in Human Behavior, 28 (2), 331-339.

Leung, L., \& Wei, R. (2000). More than just talk on the move: Uses and gratifications of the cellular phone. Journal of Marketing Communication Quarterly, 72(2), 308-320.

Liao, Y., Wang, C., Huang, Y., \& Su, Z. (2016). Building a Facebook embedded picture book design learning platform and understanding its use intentions. Paper presented at the Pacific Asia Conference on Information Systems, PACIS 2016 - Proceedings. http://aisel.aisnet. org/pacis2016/53

Luo, M.M., Chea, S., \& Chen, J.S. (2011). Web-based information service adoption: A comparison of the motivational model and the uses and gratifications theory. Decisions Support Systems, 51, 21-30. https:// doi.org/10.1016/j.dss.2010.11.015.

Luo, M.M., \& Remus, W. (2014). Uses and gratifications and acceptance of Web-based information services: An integrated model. Computers in Human Behavior, 38, 281-295.

Ma, S., Zhang, S., Li, G. \& Wu, Y. (2019), Exploring information security education on social media use: Perspective of uses and gratifications theory, Aslib Journal of Information Management, 71 (5), 618-636. https://doi.org/10.1108/AJIM-09-2018-0213

Mazman, S. G., \& Usluel, Y. K. (2010). Modeling educational use of Facebook. Computers \& Education, 55(2), 444-453. https://doi.org/10.1016/j.compedu.2010.02.008.

Mingle, J., \& Adams, M. (2015). Social media network participation and academic performance in senior high schools in Ghana. Library Philosophy and Practice, 2015(1), 1286. http://digitalcommons.unl.edu/ libphilprac/1286

Morales, A., Ortega, E., Conesa, E., \& Ruiz-Esteban, C. (2017). Análisis bibliométrico de la producción científica en Educación Musical en España. Revista Española de Pedagogía, 268, 399-414. https://doi. org/10.22550/REP75-3-2017-07

Özad, B.E., \& Uygarer, G. (2014). Attachment needs and social networking sites. Social Behavior and Per- 
sonality: an international journal, 42(1), 43-52. https:// doi.org/10.2224/sbp.2014.42.0.S43

Park, N., Lee, K.M., \& Cheong, P.H. (2007), University Instructors' Acceptance of Electronic Courseware: An Application of the Technology Acceptance Model. Journal of Computer Mediated Communication, 13, 163186. https://doi.org/10.1111/j.1083-6101.2007.00391.x

Pereira, C. A. (2018). A mídia na Ciência da Informação. Transinformação, 30(2), 141-152. http://dx.doi. org/10.1590/2318-08892018000200001

Perera, C.H., Nayak, R. \& Nguyen, L.T. (2020) Social brand engagement and brand positioning for higher educational institutions: an empirical study in Sri Lanka, Journal of Marketing for Higher Education, November 2020. https://doi.org/10.1080/08841241.20 20.1841068

Purnawarman, P., Susilawati, S., \& Sundayana, W. (2016). The use of Edmodo in teaching writing in a blended learning setting. Indonesian Journal of Applied Linguistics, 5(2), 242-252. https://doi. org/10.17509/ijal.v5i2.1348

Rathnayake, C., \& Winter, J. S. (2018). Carrying forward the uses and grats 2.0 agenda: An affordance-driven measure of social media uses and gratifications. Journal of Broadcasting and Electronic Media, 62(3), 371-389. https://doi.org/10.1080/08838151.2018.1451861

Raza, S.A., Qazi, W., Umer, B., \& Khan, K. A. (2020). Influence of social networking sites on life satisfaction among university students: A mediating role of social benefit and social overload. Health Education, 120(2), 141-164. https://doi.org/10.1108/HE-072019-0034

Saini, Ch., \& Abraham, J. (2019). Modeling educational usage of social media in pre-service teacher education. Journal of Computing in Higher Education, 31, 21-55. https://doi.org/10.1007/s12528-018-91904(0123456789

Sarapin, S.H., \& Morris, P.L. (2015). Faculty and Facebook friending: Instructor-student online social communication from the professor's perspective. The Internet and Higher Education, 27, 14-23. http://dx. doi.org/10.1016/j.iheduc.2015.04.0011096-7516/

Sheldon, P., Rauschnabel, P.A., Antony, M.G., \& Car, S. (2017). A cross-cultural comparison of croatian and american social network sites: Exploring cultural differences in motives for Instagram use. Computers in Human Behavior, 75, 643-651. https://doi. org/10.1016/j.chb.2017.06.009

Scholtz, B., Calitz, A.P., \& Tlebere, T. (2017). Evaluating students' social media use for extra-curricular educa- tion. Journal of Applied Research in Higher Education, 9(1), 5-23. https://doi.org/10.1108/JARHE-05-2015-0039

Thongsri, N., Shen, L., Bao, Y., \& Alharbi, I.M. (2018). Integrating UTAUT and UGT to explain behavioural intention to use M-learning: A developing country's perspective. Journal of Systems and Information Technology, 20(3), 278-297. https://doi.org/10.1108/JSIT11-2017-0107

Urrútia, G., \& Bonfill, X. (2010). Declaración PRISMA: una propuesta para mejorar la publicación de revisiones sistemáticas y metaanálisis. Medicina Clínica, 135(11), 507-511.

Vrocharidou, A., \& Efthymiou, I. (2012). Computer mediated communication for social and academic purposes: Profiles of use and university students' gratifications. Computers \& Education, 58(1), https:// doi.org/609-616. 10.1016/j.compedu.2011.09.015

Wu, Y. \& Song, D. (2019) Gratifications for Social Media Use in Entrepreneurship Courses: Learners' Perspective. Frontiers in Psychology. 10, 12-70. https://doi. org/10.3389/fpsyg.2019.01270

Yakin, I., \& Tinmaz, H. (2015). Theoretical guidelines for the utilization of instructional social networking websites. Turkish Online Journal of Distance Education, 16(4), 67-83. https://doi.org/10.17718/tojde.81802

Yen, C. (2016). How to unite the power of the masses? exploring collective stickiness intention in social network sites from the perspective of knowledge sharing. Behaviour and Information Technology, 35(2), 118133. https://doi.org/10.1080/0144929X.2015.1105297

Yen, W., Chang, C., \& Chou, S. (2019). More than just fame: Learning from internet Celebrities-Uses and gratifications perspective. 2nd International Conference on Innovative Technologies and Learning, ICITL (pp. 407-416). https://doi.org/10.1007/978-3-030-353438_44

Zhang Y. \& Rau P. (2015) Field Study on College Students' Uses and Gratifications of Multitasking Interaction with Multiple Smart Devices. In: Rau P. (ed) Cross-Cultural Design Applications in Mobile Interaction, Education, Health, Transport and Cultural Heritage. CCD 2015. Lecture Notes in Computer Science, vol 9181. Springer, Cham. https://doi.org/10.1007/978-3-31920934-0_38

Zhou, Z., Jin, X., Vogel, D. R., Fang, Y., \& Chen, X. (2011). Individual motivations and demographic differences in social virtual world uses: an exploratory investigation in second life. International Journal of Information Management, 31(3), 261-271. https://doi. org/10.1016/j.ijinfomgt.2010 